\title{
The Emergence of Hyperchaos and Synchronization in Networks with Discrete Periodic Oscillators
}

\author{
Adrian Arellano-Delgado ${ }^{1}$, Rosa Martha López-Gutiérrez ${ }^{1}$, Miguel Angel Murillo-Escobar ${ }^{2}$, \\ Liliana Cardoza-Avendaño ${ }^{1}$ and César Cruz-Hernández ${ }^{2, *}$ \\ 1 Facultad de Ingeniería Arquitectura y Diseño, Universidad Autónoma de Baja California (UABC), \\ (CONACYT-UABC), Ensenada BC 22860, Mexico; adrian.arellano@uabc.edu.mx (A.A.-D.); \\ roslopez@uabc.edu.mx (R.M.L.-G.); lcardoza@uabc.edu.mx (L.C.-A.) \\ 2 Electronics and Telecommunications Department, Scientific Research and Advanced Studies Center of \\ Ensenada (CICESE), Ensenada BC 22860, Mexico; mmurillo@cicese.mx \\ * Correspondence: ccruz@cicese.mx; Tel.: +52-646-175-0500
}

Received: 9 May 2017; Accepted: 8 August 2017; Published: 16 August 2017

\begin{abstract}
In this paper, the emergence of hyperchaos in a network with two very simple discrete periodic oscillators is presented. Uncoupled periodic oscillators may represent, in the crudest and simplest form, periodic oscillators in nature, for example fireflies, crickets, menstrual cycles of women, among others. Nevertheless, the emergence of hyperchaos in this kind of real-life network has not been proven. In particular, we focus this study on the emergence of hyperchaotic dynamics, considering that these can be mainly used in engineering applications such as cryptography, secure communications, biometric systems, telemedicine, among others. In order to corroborate that the emerging dynamics are hyperchaotic, some chaos and hyperchaos verification tests are conducted. In addition, the presented hyperchaotic coupled system synchronizes, based on the proposed coupling scheme.
\end{abstract}

Keywords: emergence of hyperchaos; complex dynamical systems; network synchronization; periodic oscillators; entropy

\section{Introduction}

The emergence of chaos by coupling two or more systems is a widely-observed phenomenon that has attracted the attention of the scientific community for decades. Particularly, two procedures for the emergence of chaos or hyperchaos by coupling two or more systems have been studied. In the first instance, we have the case of coupled chaotic oscillators in the periodic regime; see for example [1-3]. The second case is by means of the coupling of non-chaotic oscillators $[4,5]$.

In the current literature, the majority of the research works concerning the emergence of chaos in networks consider uncoupled chaotic nodes in the periodic regimen, that is chaotic nodes with parameter values in non-chaotic regions. Then, these nodes are coupled by using different network topologies. As a result, all nodes may be able to transit to a chaos state; see, e.g., [6-8]. In addition, to our knowledge, relative to the emergence of chaos and hyperchaos in networks, previous works consider rings of three or more unidirectionally-coupled oscillators; see, e.g., $[9,10]$.

On the other hand, the synchronization of complex networks has received great interest in different fields of science and technology, in particular, synchronization of complex dynamical networks with chaotic systems as nodes; see, e.g., [11-14]. Interaction among coupled nodes within a complex network plays an important role in the emerging dynamics of networks, for example synchronization or emergence of chaos [6].

At present, special attention is paid to the study of complex networks in nature and the phenomena arising in them [15-17]. The study of complex networks' synchronization is of great interest in the 
scientific community, with similar behaviors present in natural networks, for example communities of fireflies, crickets, menstrual cycles of women, among others.

Recently, we have studied the coupling of periodic oscillators, as a very simple model of fireflies on nearest-neighbor, star and small-world topologies [18]. This allows understanding some important aspects of behavior, interaction, coupling and synchronization of simple models that can represent living organisms with common goals and collective behavior.

The case of hyperchaos emergence with coupled non-chaotic oscillators is presented in this paper, where the entropy-that describes the dispersal of a system's energy and is an indication of the disorder of a physical system [19-21], appear with the coupling of the simple periodic oscillators, i.e., high entropy is obtained from a coupling of systems with very low entropy. In addition, we present the peculiarity that along with the emergence of hyperchaos, with particular configuration and parameters, we achieve hyperchaotic synchronization (in-phase or anti-phase) of discrete periodic oscillators that is based on the complex systems theory.

The main contribution of this paper is the emergence of chaos or hyperchaos making a coupling of only two very simple periodic discrete systems, which in the crudest and simplest form can represent real-life periodic oscillators, like the ones mentioned above, that in isolation in no way generate chaos or hyperchaos, i.e., structurally non-chaotic. These emergent hyperchaotic dynamics can be mainly used in information theory [22], in engineering applications such as cryptography, secure communications, biometric systems, telemedicine, among others; see, e.g., [23-25]. Given the simplicity and discrete nature of the proposed simple network, it may be attractive for digital implementations by using embedded systems such as Field Programmable Gate Arrays (FPGAs), Digital Signals Processors (DSPs) or microcontrollers; see, e.g., [26].

This paper is organized as follows. In Section 2, some basic concepts on the synchronization of complex dynamical systems are presented. In Section 3, the emergence of hyperchaos in a simple network from the interaction of only two discrete periodic oscillators that are bidirectionally coupled is studied. In Section 4, some tests that confirm hyperchaos emergence are presented. In Section 5, we show the synchronization of two bidirectionally-coupled hyperchaotic nodes. Finally, in Section 6, some conclusions are presented.

\section{Complex Dynamical Networks}

We consider a complex dynamical network composed of $N$ identical nodes, linearly and diffusively coupled through the first state of each node. In this dynamical network, each node constitutes an $n$-dimensional discrete-time map. The state equations of this network are described by:

$$
\mathbf{x}_{i}(k+1)=f\left(\mathbf{x}_{i}(k)\right)+\mathbf{u}_{i}(k), i=1,2, \ldots, N,
$$

where $\mathbf{x}_{i}(k)=\left(x_{i 1}(k), x_{i 2}(k), \ldots, x_{i n}(k)\right)^{T} \in \mathbb{R}^{n}$ are the state variables of the node $i$, $\mathbf{u}_{i}(k)=\left(u_{i 1}(k), 0, \ldots, 0\right)^{T} \in \mathbb{R}^{n}$ is the input signal of the node $i$ and is defined by:

$$
\mathbf{u}_{i}(k)=c \sum_{j=1}^{N} a_{i j} \Gamma \mathbf{x}_{j}(k), \quad i=1,2, \ldots, N,
$$

the constant $c>0$ represents the coupling strength of the complex network, and $\Gamma \in \mathbb{R}^{n \times n}$ is a constant 0-1 matrix linking coupled state variables; whereas, $\mathbf{A}=\left(a_{i j}\right) \in \mathbb{R}^{N \times N}$ is the coupling matrix, which represents the coupling topology of the complex network. If there is a connection between node $i$ and node $j$, then $a_{i j}=1$; otherwise, $a_{i j}=0$ for $i \neq j$. The diagonal elements of coupling matrix $\mathbf{A}$ are defined as:

$$
a_{i i}=-\sum_{j=1, j \neq i}^{N} a_{i j}=-\sum_{j=1, j \neq i}^{N} a_{j i}, i=1,2, \ldots, N .
$$

If the degree of node $i$ is $d_{i}$, then $d_{i}=-a_{i i}, i=1,2, \ldots, N$. 
Now, suppose that the complex network (1) and (2) are connected without isolated clusters. Then, $\mathbf{A}$ is a symmetric irreducible matrix. In this case, it can be shown that zero is an eigenvalue of $\mathbf{A}$ with multiplicity one, and all of the other eigenvalues of $\mathbf{A}$ are strictly negative; see $[27,28]$.

In accordance with [28] for discrete systems, the complex dynamical network (1) and (2) are said to achieve (asymptotically) synchronization if:

$$
\mathbf{x}_{1}(k)=\mathbf{x}_{2}(k)=, \ldots,=\mathbf{x}_{N}(k) \text {, as } k \rightarrow \infty .
$$

The diffusive coupling condition (3) guarantees that the synchronization state is a solution, $\mathbf{s}(k) \in \mathbb{R}^{n}$, of an isolated node, that is:

$$
\mathbf{s}(k+1)=f(\mathbf{s}(k)),
$$

where $\mathbf{s}(k)$ can be an equilibrium point, a periodic orbit or, a chaotic attractor. Thus, the stability of the synchronization state,

$$
\mathbf{x}_{1}(k)=\mathbf{x}_{2}(k)=, \ldots,=\mathbf{x}_{N}(k)=\mathbf{s}(k),
$$

of the complex network (1) and (2) are determined by the dynamics of an isolated node, i.e., function $f$ and solution $\mathbf{s}(k)$, the coupling strength $c$, the inner linking matrix $\Gamma$ and the coupling matrix $\mathbf{A}$.

\section{Emergence of Hyperchaos with Coupled Periodic Oscillators}

This section describes the state equations for networks with bidirectionally-coupled periodic oscillators like nodes.

\subsection{Uncoupled Periodic Oscillators}

Dynamical networks with discrete periodic oscillators are constructed, which in a simple way, may represent organisms interacting with each other, for example fireflies [18]. Figure 1 illustrates a simple network with two bidirectionally-coupled discrete periodic nodes.

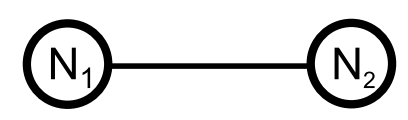

Figure 1. Simple network with two bidirectionally-coupled periodic nodes.

State equations for this dynamical network are given as follows. The first node $N_{1}$ is described by:

$$
\left\{\begin{array}{l}
w_{1}(k+1)=w_{1}(k) b+u_{11}(k), \\
x_{1}(k+1)=\sin \left(w_{1}(k) t(k)\right),
\end{array}\right.
$$

with input signal:

$$
u_{11}(k)=c\left(-\eta x_{1}(k)+x_{2}(k)\right)
$$

the second node $N_{2}$ is given by:

$$
\left\{\begin{array}{l}
w_{2}(k+1)=w_{2}(k) b+u_{21}(k), \\
x_{2}(k+1)=\sin \left(w_{2}(k) t(k)\right),
\end{array}\right.
$$

with input signal:

$$
u_{21}(k)=c\left(-\eta x_{2}(k)+x_{1}(k)\right),
$$

and the time step is given by:

$$
t(k+1)=t(k)+\frac{\pi}{1000} .
$$


Note that in the network with two coupled periodic discrete nodes (7)-(11), the parameters $\eta$ and $b$ are introduced, with the purpose to control the interaction between the periodic oscillators. Therefore, the appropriate choice of these parameter values determines whether the emerging collective dynamics in the network are periodic, chaotic or hyperchaotic. Let us consider the following particular parameter values $\eta=-0.5$ and $b=1$ and initial conditions: $w_{1}(0)=2 \pi 2, w_{2}(0)=2 \pi 5, x_{1}(0)=x_{2}(0)=0$ and $t(0)=0$. If coupling strength $c=0$, then $N_{1}$ and $N_{2}$ are uncoupled nodes, i.e., $u_{11}(k)=u_{21}(k)=0$; node $N_{1}$ corresponds to a simple periodic oscillator at $2 \mathrm{~Hz}$, and node $N_{2}$ corresponds to a simple periodic oscillator at $5 \mathrm{~Hz}$. Figure 2 shows state trajectories and error dynamics $x_{1}(k)-x_{2}(k)$ (with discrete nodes, the default interpolation of MATLAB R2014a is used, in order to better appreciate the temporary graphics); while Figure 3 exhibits the phase portrait for isolated periodic oscillators (7)-(11).
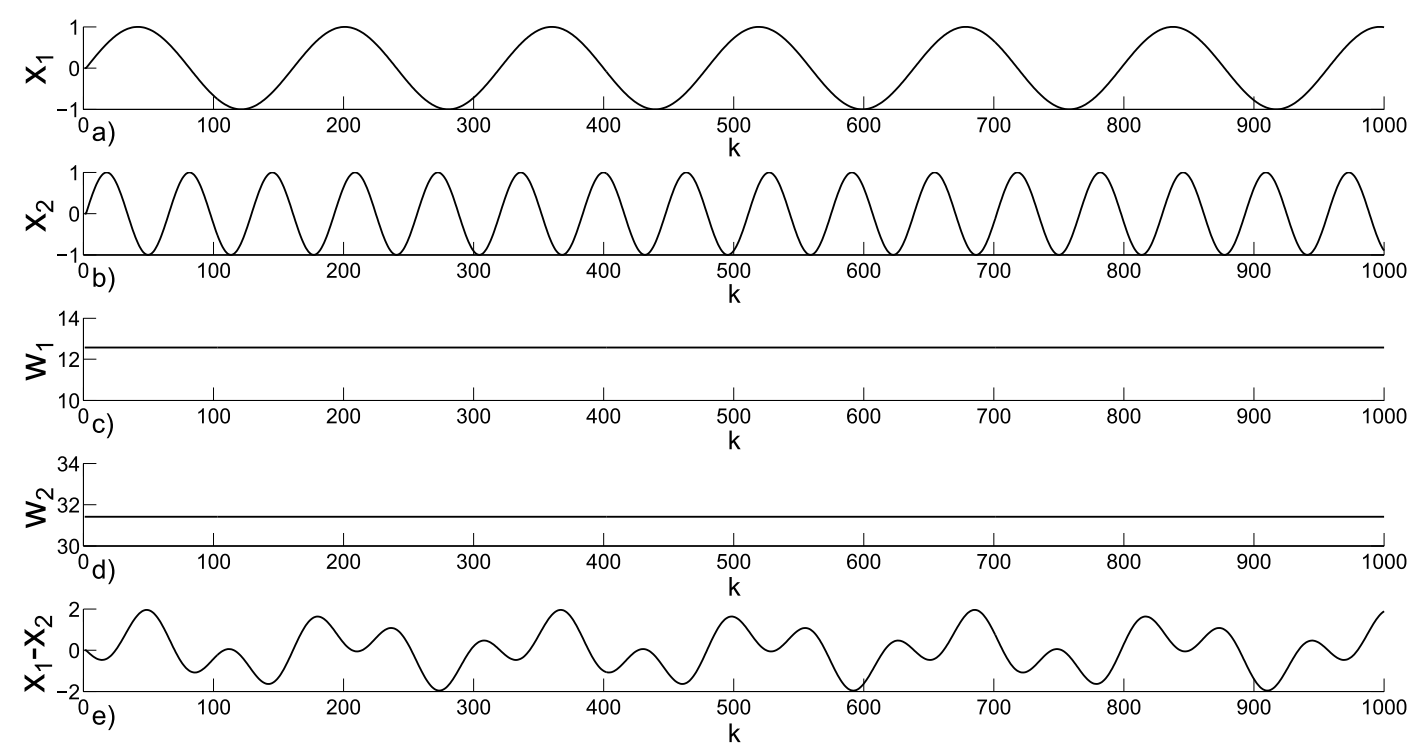

Figure 2. Temporal dynamics of states (a) $x_{1}(k)$; (b) $x_{2}(k)$; (c) $w_{1}(k)$; (d) $w_{2}(k)$; (e) $x_{1}(k)-x_{2}(k)$, with $u_{11}(k)=u_{21}(k)=0$.

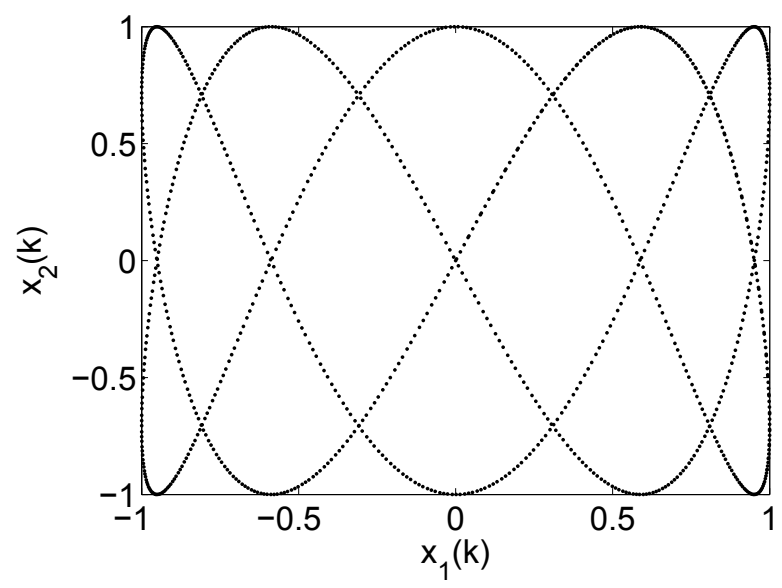

Figure 3. Phase portrait $x_{1}(k)$ versus $x_{2}(k)$ with $u_{11}(k)=u_{21}(k)=0$.

It can be seen that oscillators $N_{1}$ and $N_{2}$ have periodical behavior when they are uncoupled, and under this scenario, the oscillators do not generate chaos. Figure 4 shows the corresponding limit cycle attractors for the periodic nodes $N_{1}$ and $N_{2}$. These limit cycle attractors are obtained by delaying one sample. This was made in order to obtain attractors given that $w_{1}(k)$ and $w_{2}(k)$ are constants. 

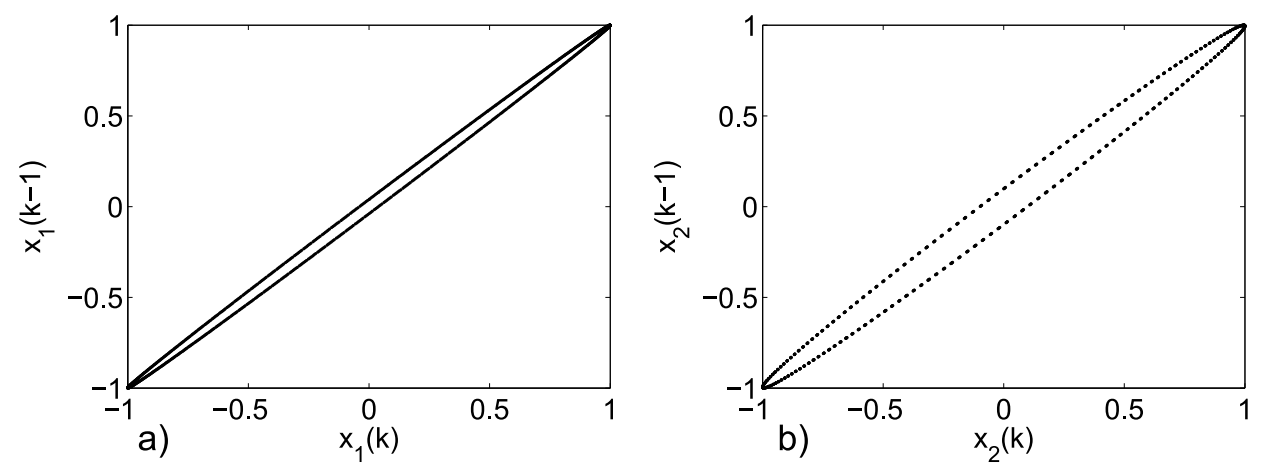

Figure 4. Limit cycle attractors generated by uncoupled periodic nodes $N_{1}$ and $N_{2}:(a) x_{1}(k)$ versus $x_{1}(k-1)$ and $(\mathbf{b}) x_{2}(k)$ versus $x_{2}(k-1)$.

\subsection{Emerging Hyperchaos with Two Coupled Periodic Oscillators}

On the other hand, if we use a coupling strength $c=1$ in Equations (7)-(11), the emerging collective behavior in the network due to the interaction of periodic oscillators is hyperchaotic (this statement will be verified in a later section). Figure 5 shows state trajectories of $x_{1}(k), x_{2}(k), w_{1}(k)$, $w_{2}(k)$ and error dynamics $x_{1}(k)-x_{2}(k)$. Figure 6 illustrates the hyperchaotic attractors $x_{1}(k)$ versus $w_{1}(k)$ and $x_{2}(k)$ versus $w_{2}(k)$; whereas, Figure 7 shows phase portraits $x_{1}(k)$ versus $x_{2}(k)$ and $w_{1}(k)$ versus $w_{2}(k)$.
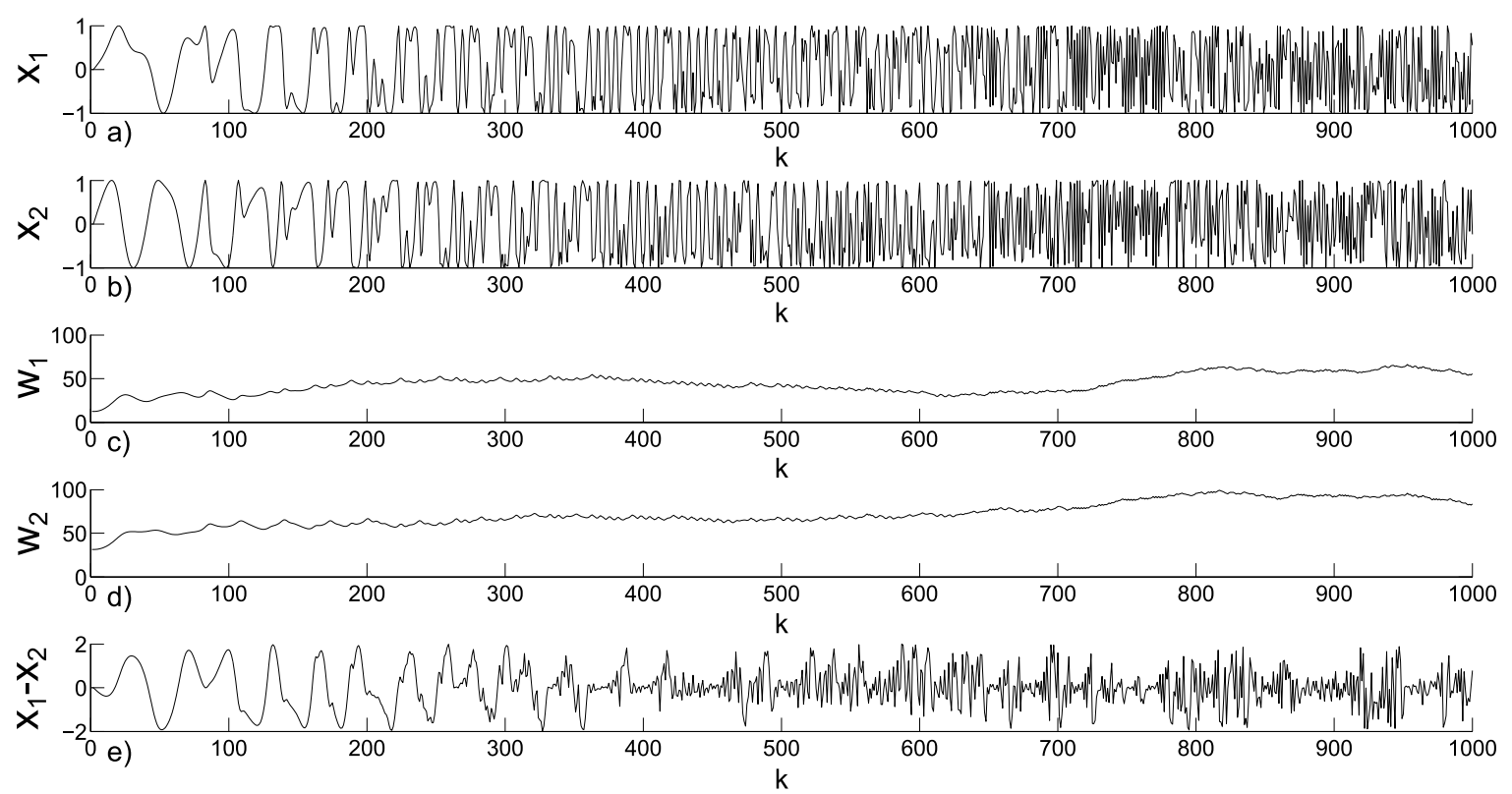

Figure 5. Time evolution for bidirectional coupling; $c=1, \eta=-0.5$ and $b=1$ : (a) $x_{1}(k) ;(\mathbf{b}) x_{2}(k)$; (c) $w_{1}(k) ;(\mathbf{d}) w_{2}(k)$ and $(\mathbf{e}) x_{1}(k)-x_{2}(k)$. 

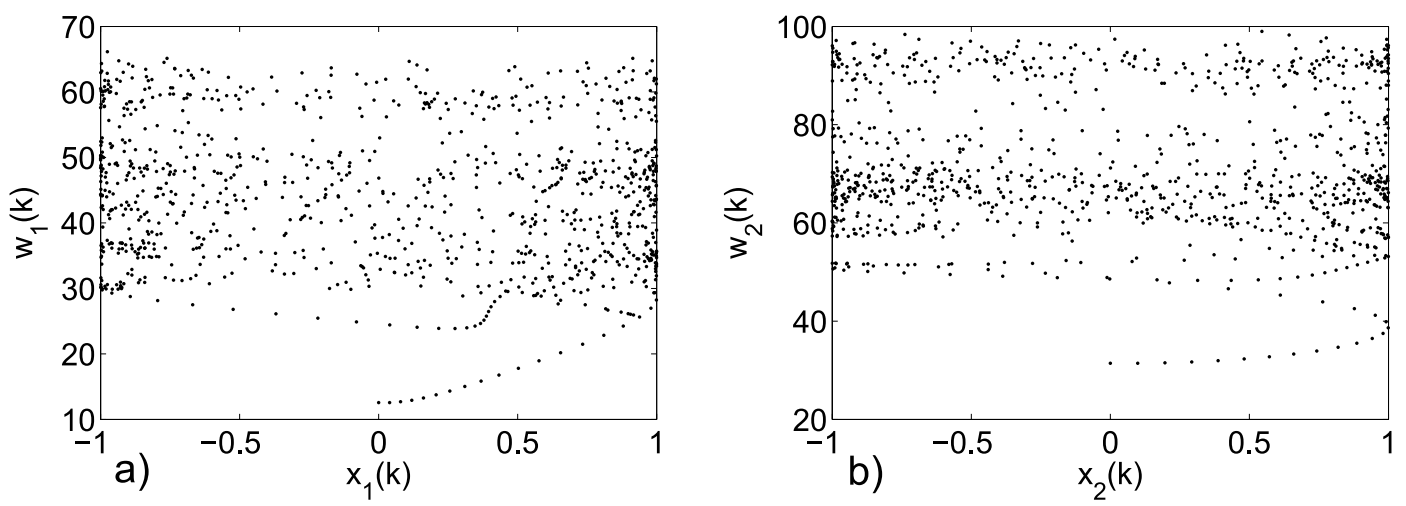

Figure 6. Hyperchaotic attractors generated by coupled nodes $N_{1}$ and $N_{2}$ : (a) $x_{1}(k)$ versus $w_{1}(k)$ and (b) $x_{2}(k)$ versus $w_{2}(k)$.
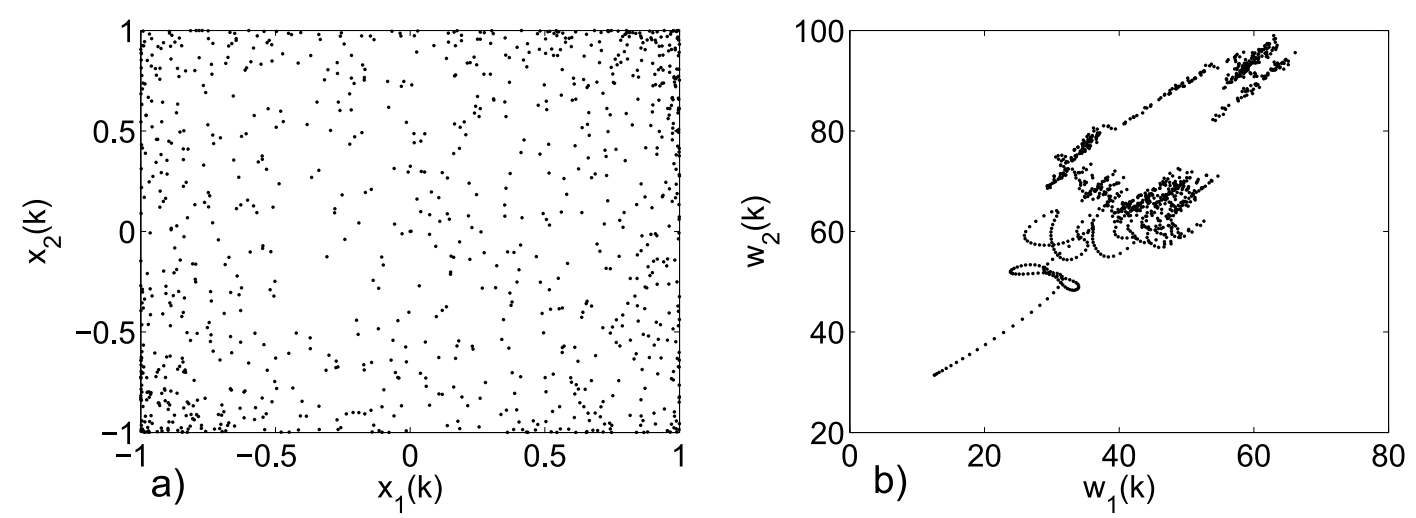

Figure 7. Phase portraits for coupled nodes $N_{1}$ and $N_{2}:(\mathbf{a}) x_{1}(k)$ versus $x_{2}(k)$ and $(\mathbf{b}) w_{1}(k)$ versus $w_{2}(k)$.

From Equations (7)-(11), we can see that the parameter $b$ directly affects the states $w_{1}(k)$ and $w_{2}(k)$, so if we change this parameter value, we can select a value of $b$ in order to have different behavior in states $w_{1}(k)$ and $w_{2}(k)$. Figure 8 a shows the bifurcation diagram for (7)-(11) of $w_{1}(k)$ with respect to parameter $b$, with $c=1, \eta=-0.5$, where the amplitude of $w_{1}(k)$ varies depending on the value of $b$; while Figure $8 \mathrm{~b}$ depicts the bifurcation diagram for state $x_{1}(k)$ with respect to parameter $b$. From Figure 8, we can establish that $|b| \leq 1$ is a sufficient condition to ensure the emergence of hyperchaos in the simple network of two coupled periodic oscillators (7)-(11). Interestingly, a peculiar behavior (like tug of war) at $b=0$ is observed; as an illustrative example, this behavior is shown in Figures 9 and 10, where we can see that $\left(x_{1}(k)\right)\left(w_{1}(k)\right)=0$ but $x_{1}(k)+w_{1}(k) \neq 0$ (the same result for states $x_{2}(k)$ and $w_{2}(k)$ is observed). Figure 11 shows the bifurcation diagram for parameter $c$, where we can see that the amplitude of $x_{1}(k)$ remains constant with the exception of values of $c$ approaching zero; on the other hand, the amplitude of $w_{1}(k)$ increases as $c$ tends to $\pm \infty$. 

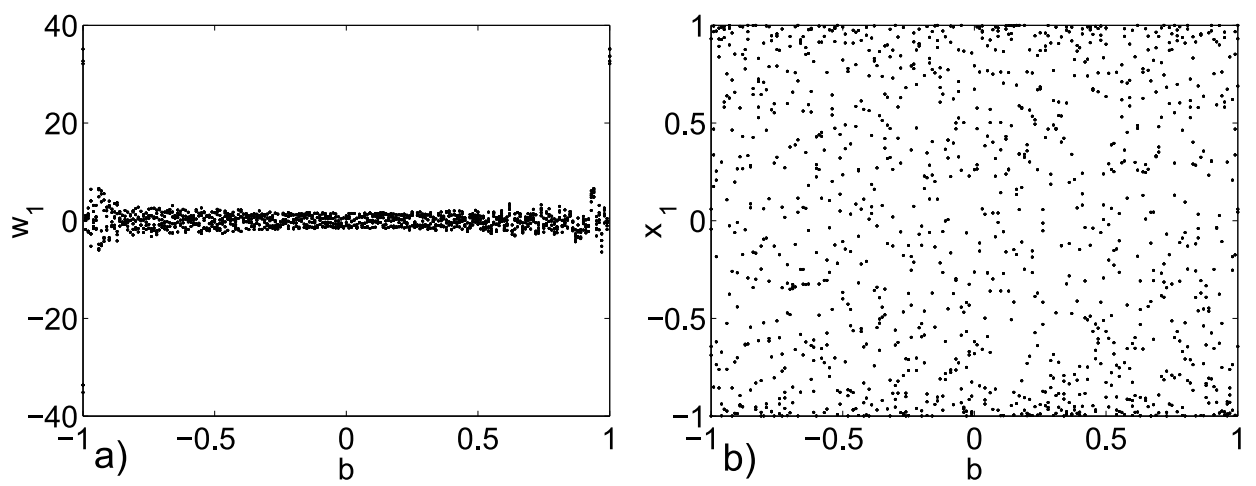

Figure 8. Bifurcation diagram of $b$ with $c=1, \eta=-0.5$, (a) $w_{1}(k)$ and (b) $x_{1}(k)$.
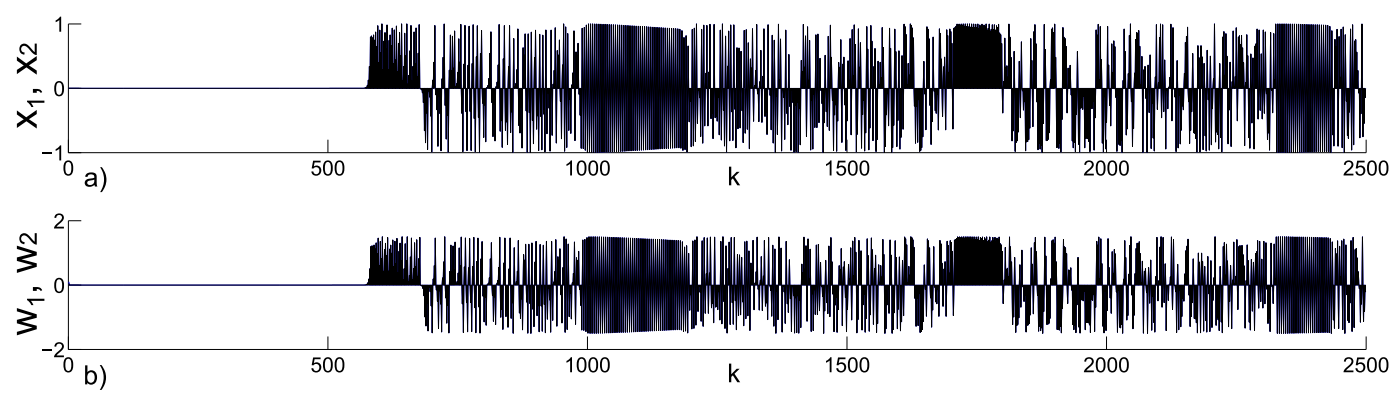

Figure 9. A peculiar collective behavior at $b=0$ for $(\mathbf{a}) x_{1}(k), x_{2}(k)$ and $(\mathbf{b}) w_{1}(k), w_{2}(k)$ (note that one iteration of the transient is suppressed).
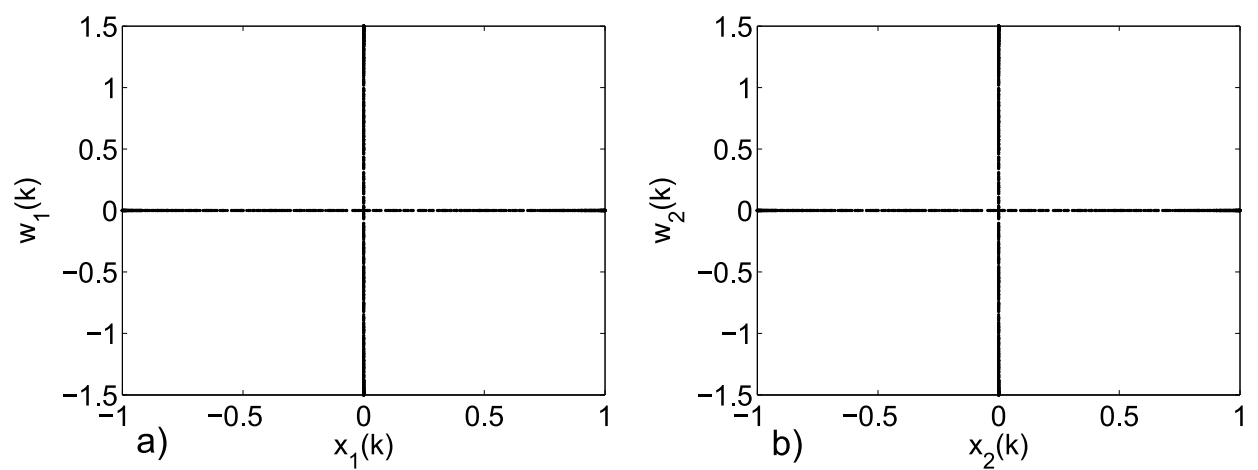

Figure 10. A peculiar collective behavior at $b=0$ for $(\mathbf{a}) x_{1}(k)$ versus $w_{1}(k)$ and $(\mathbf{b}) x_{2}(k)$ versus $w_{2}(k)$ (note that one iteration of the transient is suppressed).
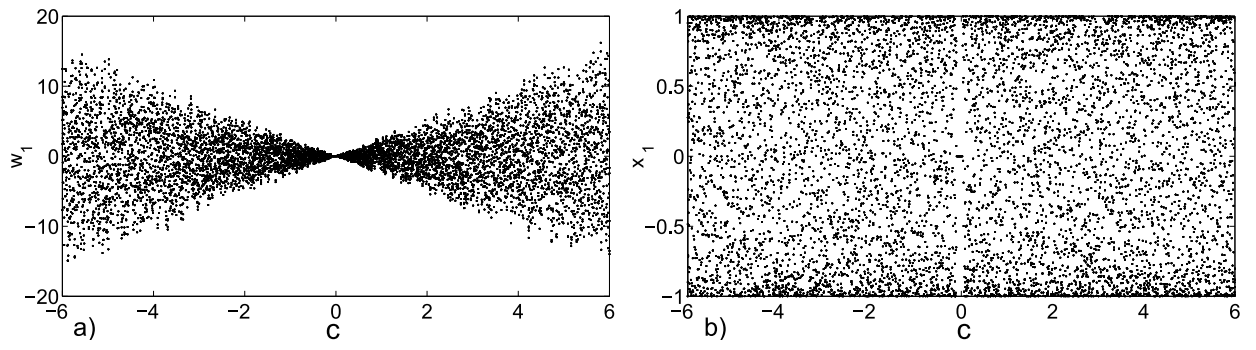

Figure 11. Bifurcation diagram of $c$ with $b=0.5, \eta=-0.5$, (a) $x_{1}(k)$ and (b) $w_{1}(k)$. 
For the case in which nodes $N_{1}$ and $N_{2}$ are unidirectionally coupled, i.e., taking (7)-(11) with $u_{11}(k)=0$, master node $N_{1}$ does not exhibit hyperchaotic dynamics. In the slave node $N_{2}$, which is influenced by the master node $N_{1}$, hyperchaotic dynamics occur; see Figure 12 . The value of $k$ now is 2500 in order to appreciate a wide range of time evolution.

Note that this study is not limited to only two coupled discrete periodic nodes; complex networks can be used to coupled more than two nodes in different topologies, such as star, nearest-neighbor, small-world, among others.
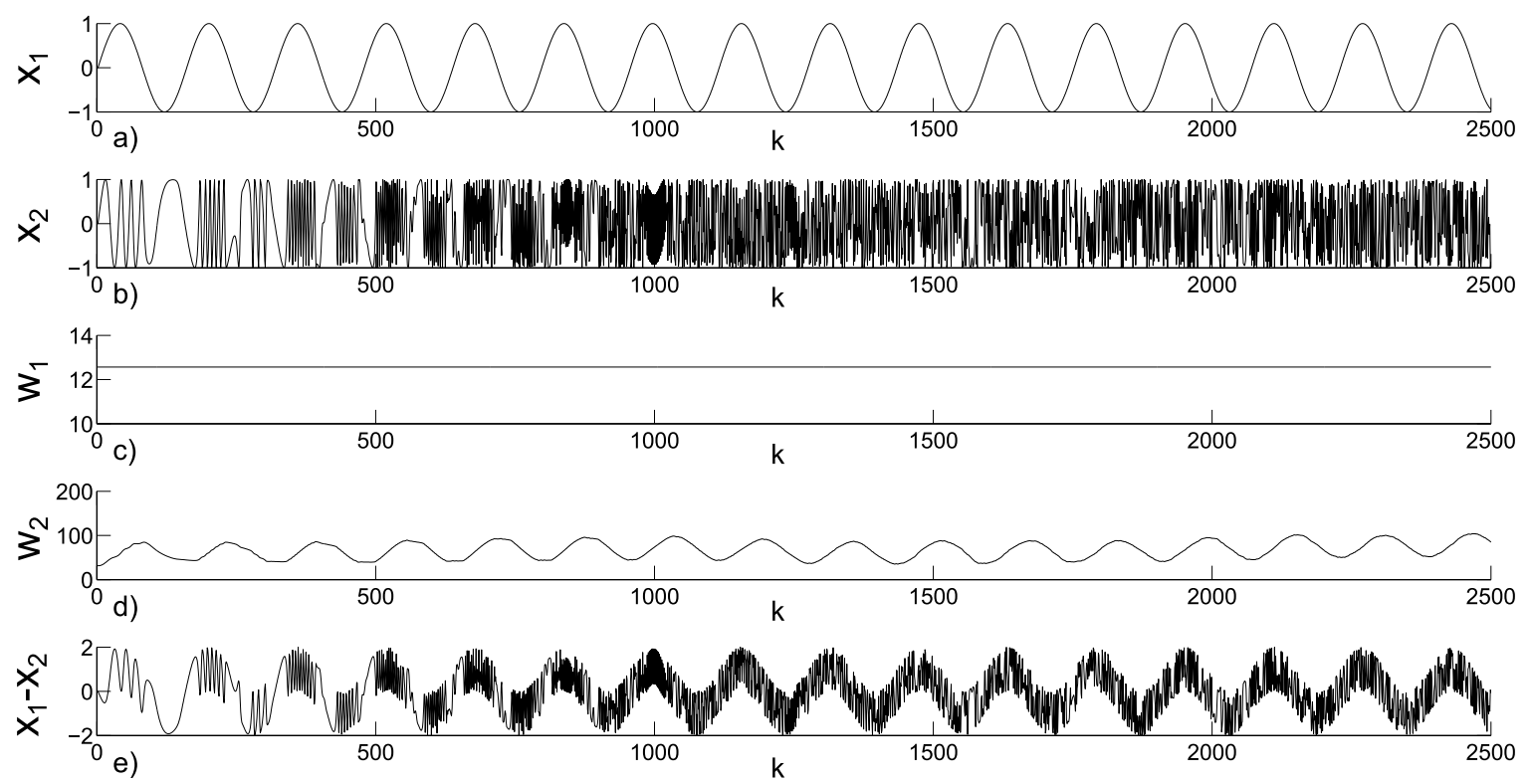

Figure 12. Time evolution for unidirectional coupling: (a) $x_{1}(k) ;(\mathbf{b}) x_{2}(k) ;(\mathbf{c}) w_{1}(k) ;(\mathbf{d}) w_{2}(k)$ and (e) $x_{1}(k)-x_{2}(k)$.

In the next section, we will carry out some tests to confirm that the emerging collective behavior in the network (7)-(11) is hyperchaos.

\section{Confirmation of Collective Hyperchaotic Behavior}

In order to verify whether the emerging collective behavior in a network with two coupled discrete periodic oscillators is chaotic or hyperchaotic, some well-known test are reported in this section: sensitivity to initial conditions, auto-correlation test, Gottwald-Melbourne 0-1 test (indicates if the signal is or is not chaotic), Lyapunov exponents (indicate chaos or hyperchaos) and, finally, the Kaplan-Yorke dimension. In the previous section, we can see that Figure 6 shows the first sign of a chaotic signal because the attractors do not have a definite shape or a limit cycle indicating periodic signals. One of the simplest, but essential tests for verification of hyperchaos is by simple visual inspection of time series with respect to sensitivity to initial conditions. Consider the network (7)-(11), with different initial conditions $w_{1}(0)=2 \pi 2, w_{2}(0)=2 \pi 5, x_{1}(0)=x_{2}(0)=0, w_{1}^{\prime}(0)=2 \pi 2.0000001$, $w_{2}^{\prime}(0)=2 \pi 5, x_{1}^{\prime}(0)=x_{2}^{\prime}(0)=0, t(0)=0, c=1, b=0.5$ and $\eta=-1$. In Figure 13, we can appreciate high sensitivity to initial conditions in the network (7)-(11). 

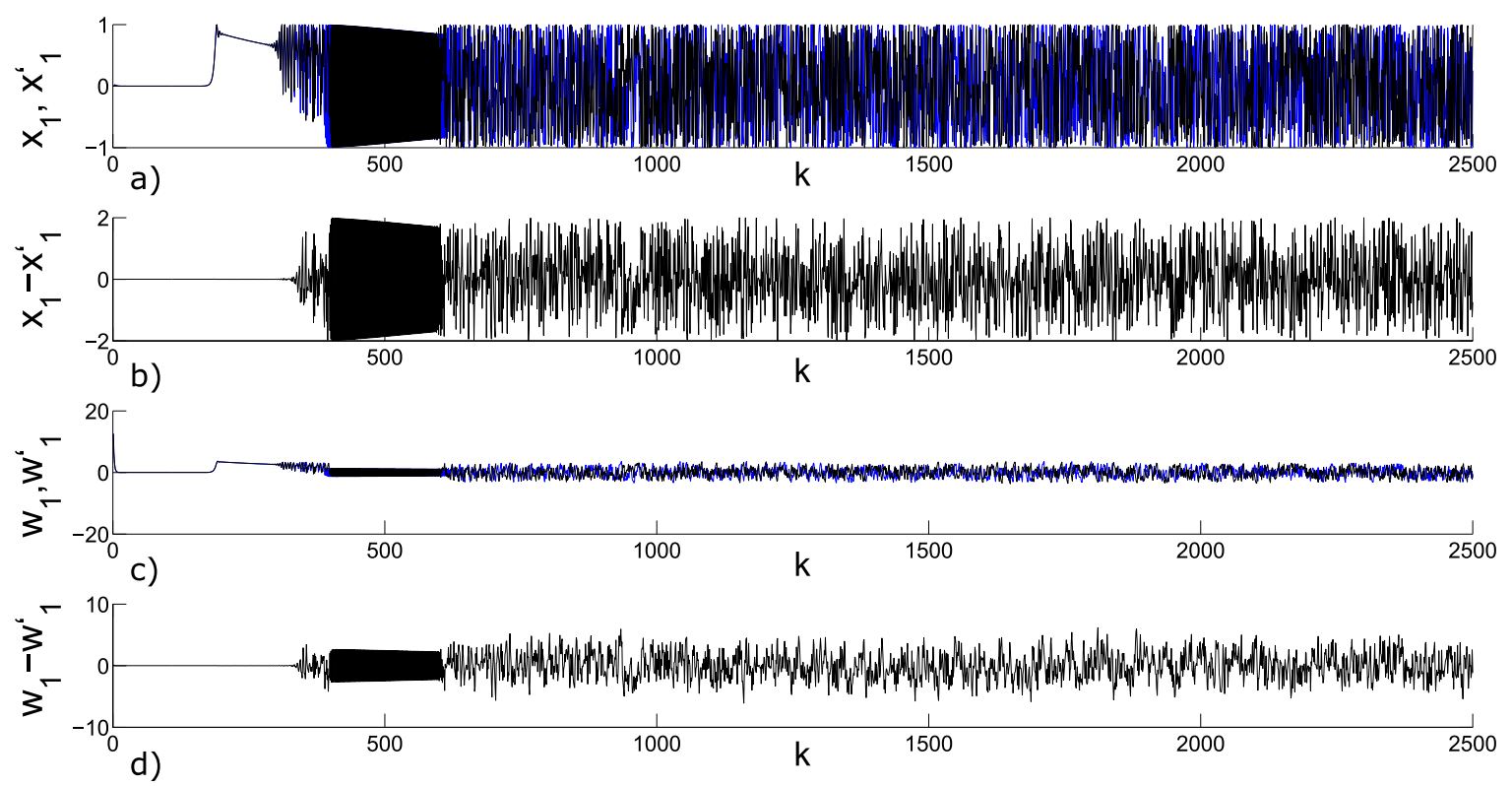

Figure 13. High sensitivity to initial conditions: (a) $x_{1}(k), x_{1}^{\prime}(k) ;(\mathbf{b}) x_{1}(k)-x_{1}^{\prime}(k) ;(\mathbf{c}) w_{1}(k) ; w_{1}^{\prime}(k)$ and (d) $w_{1}(k)-w_{1}^{\prime}(k)$.

Figure 14 shows the normalized auto-correlation of $x_{1}(k)$, which exhibits a strong peak in displacement 2500 , indicating that this point is the only one where the signal $x_{1}(k)$ presents a repeating pattern, which is clearly comparable to the auto-correlation of a quasi-random signal, that is having high entropy.

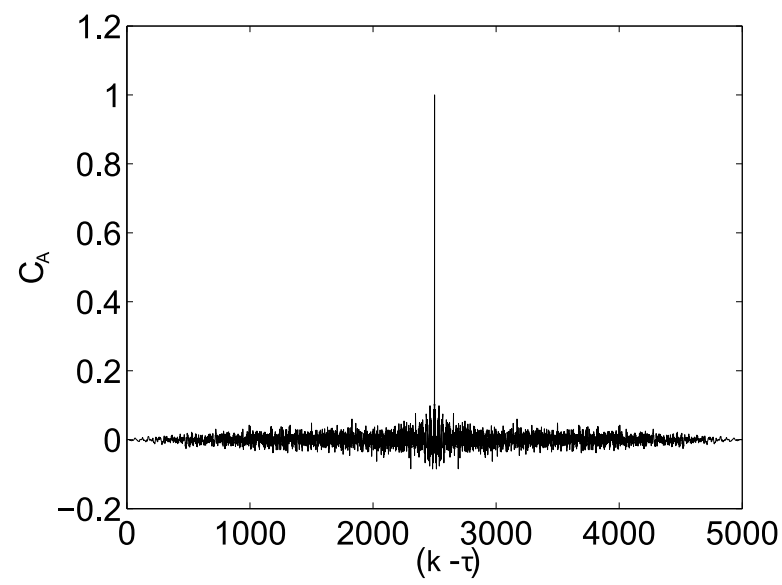

Figure 14. Auto-correlation for $x_{1}(k)$, where $C_{A}$ is the normalized auto-correlation coefficient.

Figure 15 shows the $0-1$ Gottwald-Melbourne test [29], where, if the value of $K$ is approximately zero, the analyzed signal is not chaotic ( $K$ is the obtained value from the iterative process of the Gottwald-Melbourne test), while if $K \approx 1$, the signal is chaotic. The mean value in the Gottwald-Melbourne test for signal $x_{1}(k)$ and $x_{2}(k)$ is $K_{1}=0.9968$ and $K_{2}=0.9981$, respectively, indicating that the analyzed signals are chaotic. 

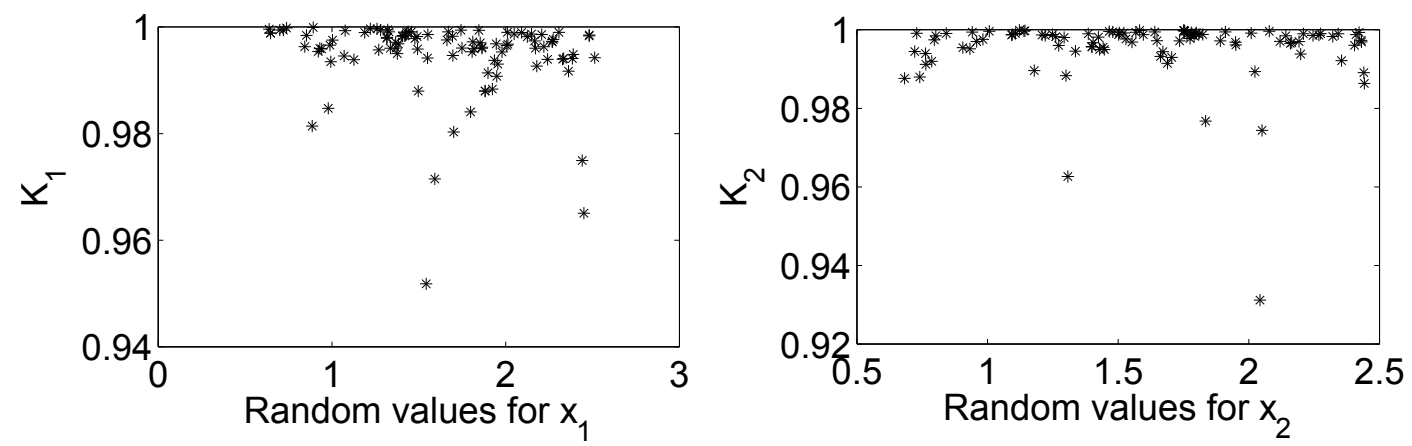

Figure 15. Gottwald-Melbourne test for signals $x_{1}(k)$ and $x_{2}(k)$.

In addition, we obtained the Lyapunov exponents of the network (7)-(11). Figure 16 shows the Lyapunov exponents $\left(L_{1}=3.11659, L_{2}=2.8843, L_{3}=0, L_{4}=-0.725755\right.$ and $\left.L_{5}=-32.575\right)$ for the network (7)-(11), wherein if at least one Lyapunov exponent is positive, then the network (7)-(11) is chaotic. Due to the network having two positive exponents, the collective behavior in the network (7)-(11) is hyperchaotic. Various kinds of fractal dimensions can be estimated theoretically and empirically, as the Hausdorff dimension, Minkowski-Bouligand dimension, box-counting dimension, correlation dimension, Kaplan-Yorke dimension, etc.; see [30-32]. The Kaplan-Yorke dimension (calculated for a time series of 350,000 iterations) for the proposed network is given by:

$$
D_{K Y}=4+\frac{L_{1}+L_{2}+L_{3}+L_{4}}{\left|L_{5}\right|}=4.1619 .
$$

The Lyapunov exponents and Kaplan-Yorke dimension were obtained by using the algorithm reported in [31].

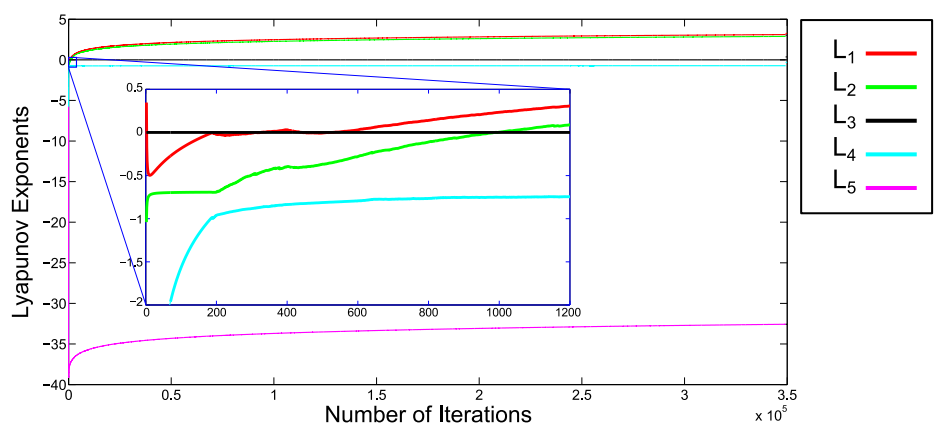

Figure 16. Lyapunov exponents for the simple network (7)-(11).

Finally, in order to determine the regions where the network (7)-(11) exhibits the emergence of hyperchaos, we construct a diagram as a function of parameters $c$ and $\eta$ in Figure 17. The diagram indicates no chaos in the green region, transition to hyperchaos in red regions and hyperchaos in blue regions. 


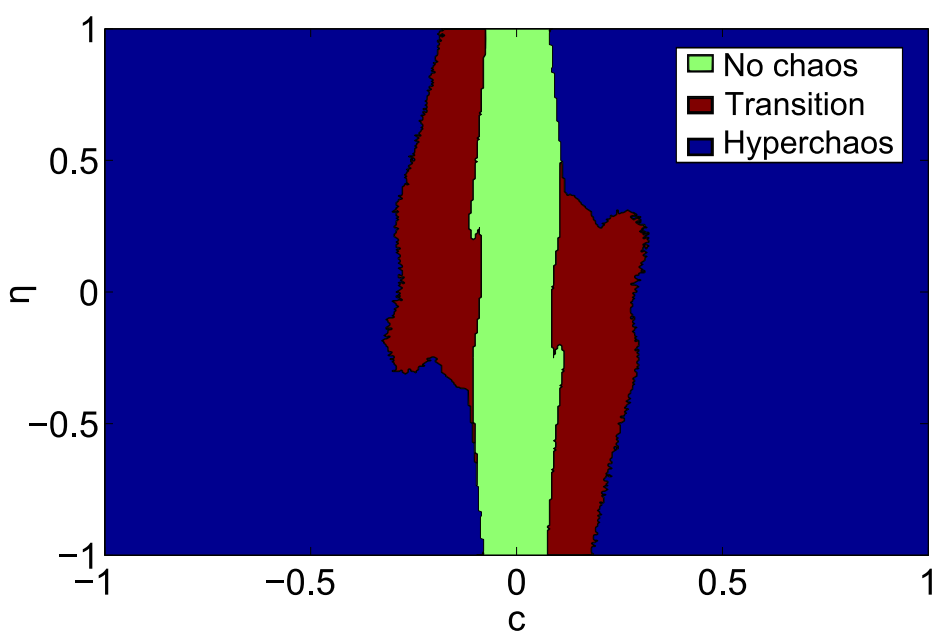

Figure 17. Diagram of hyperchaos emergence: no chaos (green), transition to hyperchaos (red) and hyperchaos (blue).

We use a 0.05 sweep step of $c$ and $\eta, k=5000$ and $b=0.5$ to obtain the hyperchaos emergence diagram of Figure 17.

In the next section, we intend to synchronize the network with two hyperchaotic nodes.

\section{Hyperchaotic Network Synchronization of Two Oscillators}

In this section, network synchronization with two bidirectionally-coupled hyperchaotic nodes $N_{1}$ and $N_{2}$, based on complex system theory, is presented.

Consider the single network (7)-(11) with parameter values $c=1, \eta=-0.5$ and $b=0.5$. Phase synchronization is achieved between the hyperchaotic nodes. As we can see in Figure 18, state trajectories and errors $x_{1}(k)-x_{2}(k)$ and $w_{1}(k)-w_{2}(k)$ are shown. Figures 19 and 20 show hyperchaotic attractors and phase portraits between two oscillators respectively. We eliminated the first five iterations in order to ignore the transitory, so that the presented simulation results in Figures 19 and 20 can be seen in more detail.
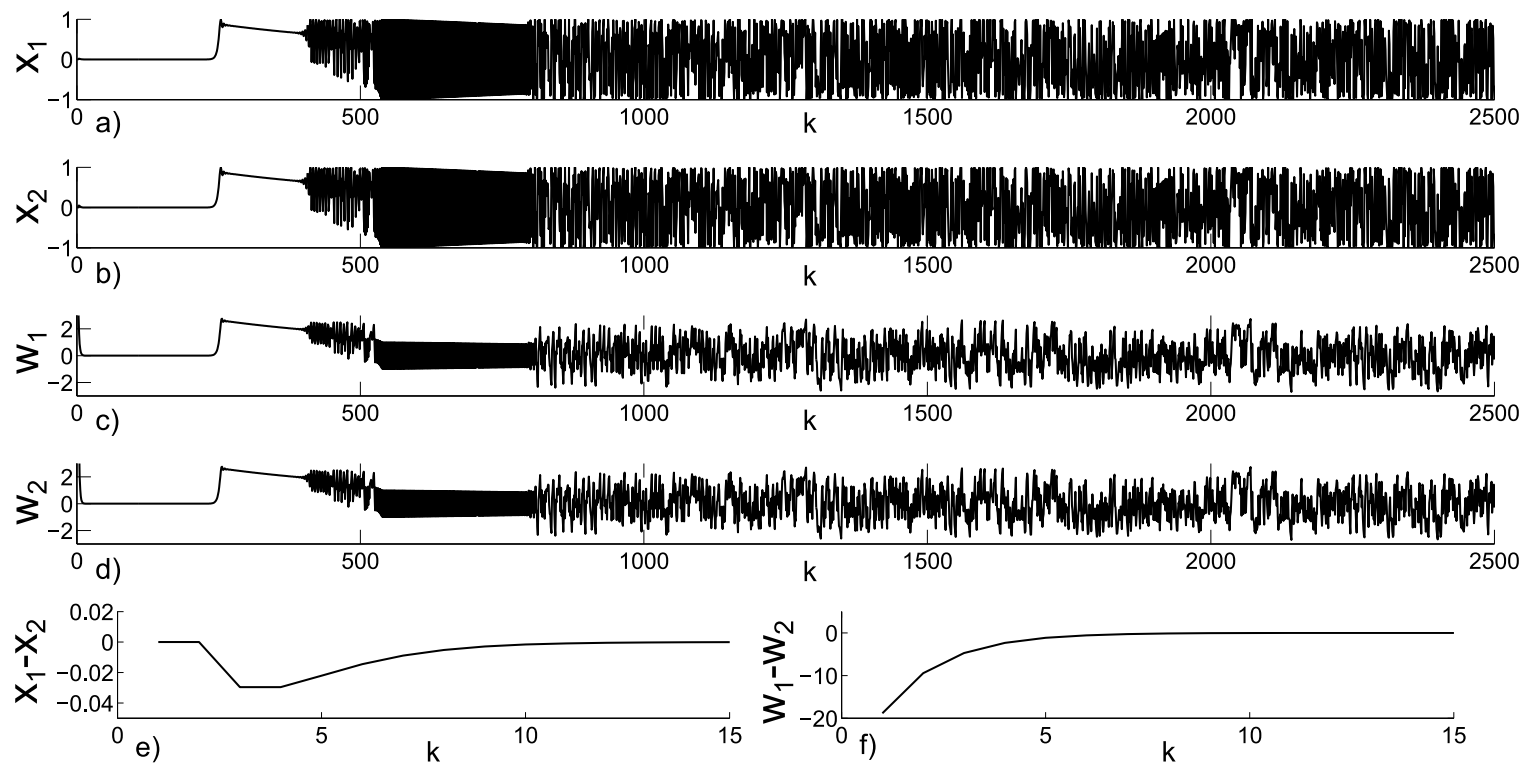

Figure 18. Time evolution for $c=1, \eta=-0.5$ and $b=0.5$, (a) $x_{1}(k)$; (b) $x_{2}(k)$; (c) $w_{1}(k)$; (d) $w_{2}(k)$; (e) $x_{1}(k)-x_{2}(k)$ and $(\mathbf{f}) w_{1}(k)-w_{2}(k)$. 

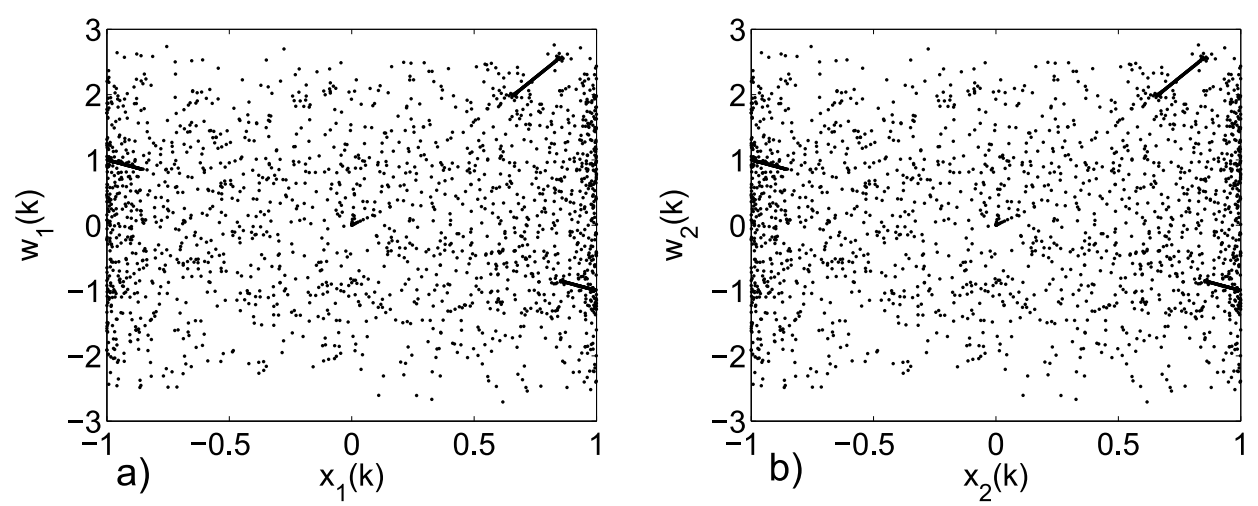

Figure 19. Hyperchaotic attractors, (a) $x_{1}(k)$ versus $w_{1}(k)$ and $(\mathbf{b}) x_{2}(k)$ versus $w_{2}(k)$ (after five iterations).
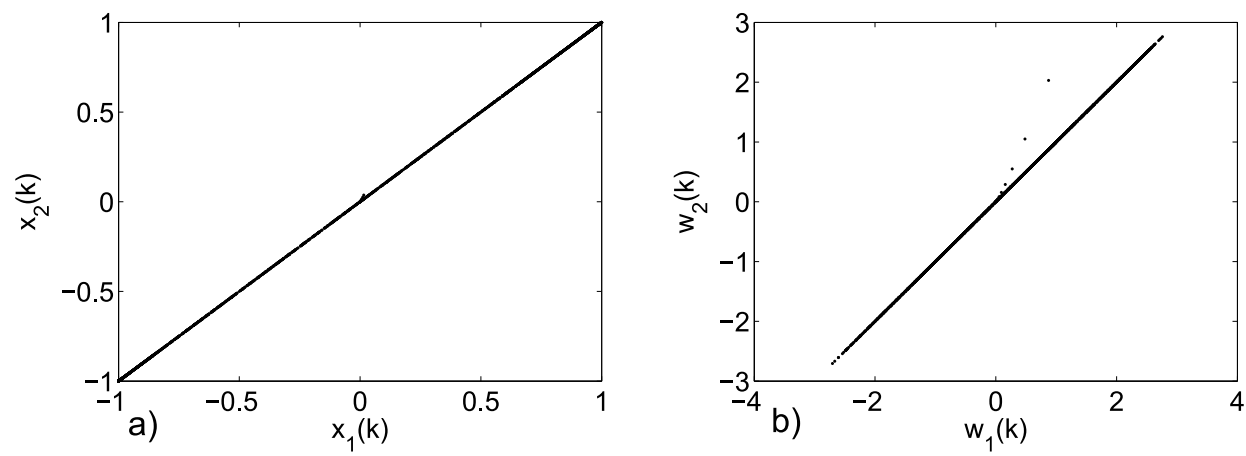

Figure 20. Phase portraits, (a) $x_{1}(k)$ versus $x_{2}(k)$ and (b) $w_{1}(k)$ versus $w_{2}(k)$ (after five iterations).

A numerical calculation for the network synchronization was performed for $-6 \leq \eta \leq 6$ at intervals of 0.001 (with $k=0,1, \ldots, 2500$, so the calculation is approximated). After removing the first 2000 iterations of each state, we review $x_{1}(k)-x_{2}(k)$ (phase synchronization) and $x_{1}(k)+x_{2}(k)$ (anti-phase synchronization). If $\left|x_{1}(k) \pm x_{2}(k)\right| \geq 0.01$ (1\% peak amplitude of $\left.x_{1}(k)\right)$, then we establish no hyperchaotic synchronization among nodes of the network (7)-(11). If $\left|x_{1}(k)-x_{2}(k)\right|<0.01$, we establish phase synchronization, and if $\left|x_{1}(k)+x_{2}(k)\right|<0.01$, we establish anti-phase synchronization. Figure 21 shows a hyperchaotic synchronization diagram with respect to $\eta$, where " 0 " denotes "no synchronization", " 1 " denotes "phase synchronization" and " 1 " denotes "anti-phase synchronization". From the hyperchaotic synchronization diagram (see Figure 21), we can see that an intermittent synchronization occurs in transitions from no synchronization to synchronization (and vice versa) in both the phase and anti-phase cases.

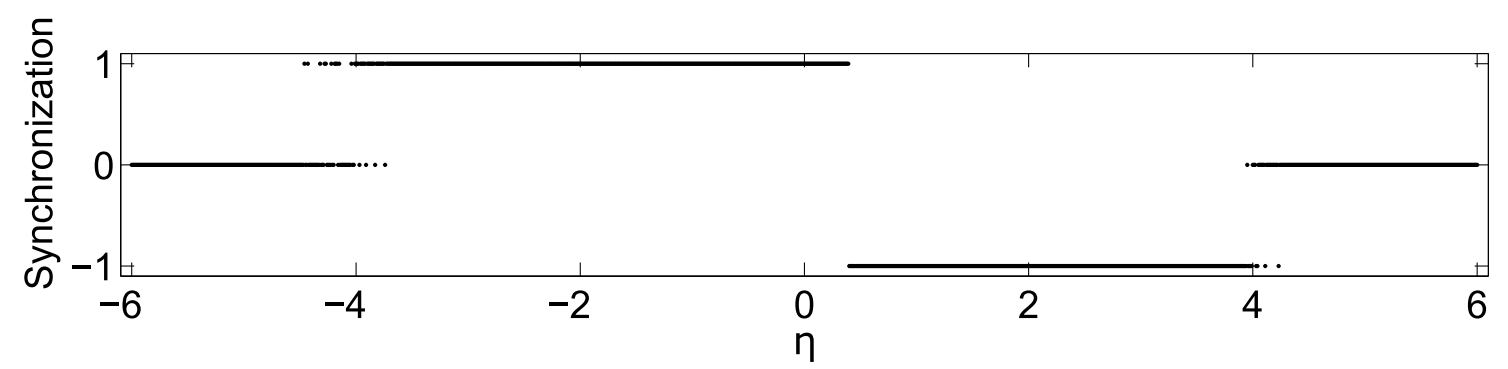

Figure 21. Hyperchaotic synchronization diagram for $\eta$ with $c=1$ and $b=0.5$.

Figures 22 and 23 show the hyperchaotic synchronization diagram of $c$ and $b$, respectively, obtained similarly as with $\eta$. 


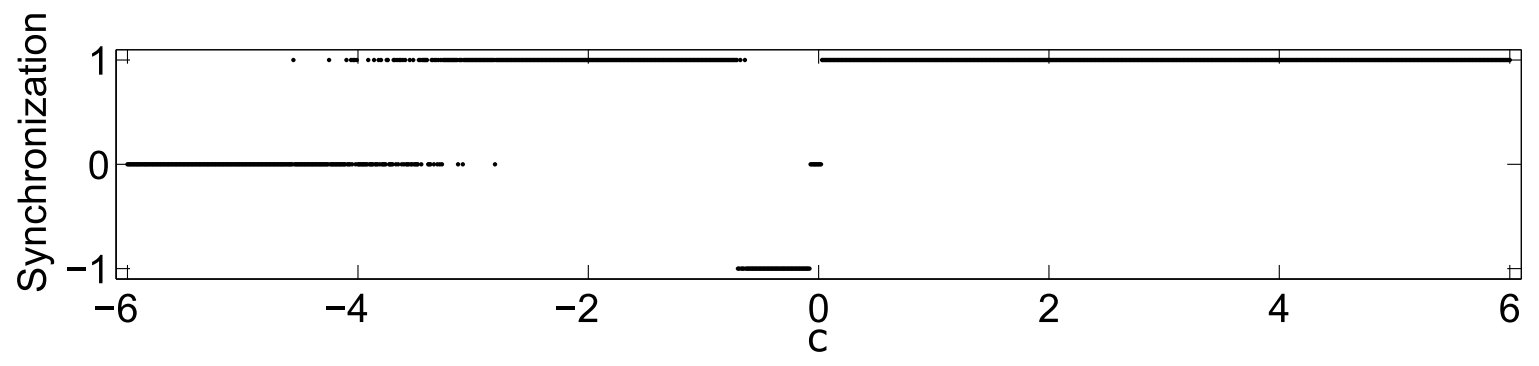

Figure 22. Hyperchaotic synchronization diagram for $c$ with $\eta=-0.5$ and $b=0.5$.

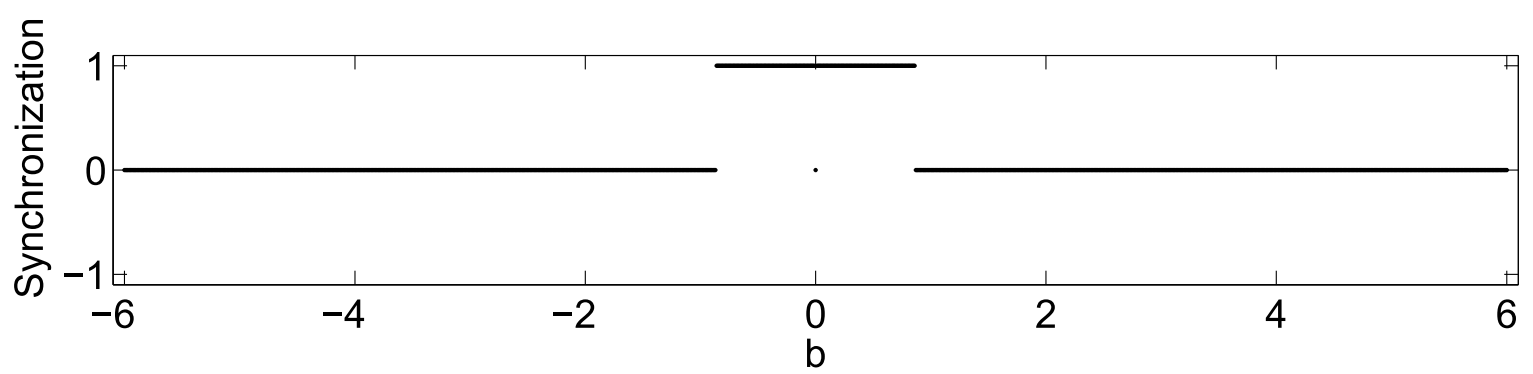

Figure 23. Hyperchaotic synchronization diagram for $b$ with $\eta=-0.5$ and $c=1$.

In Figure 24, we can appreciate the synchronization diagram of $c$ with respect to $\eta$, where, if $\eta= \pm 1$, the network synchronizes for any value of $1<c<-1$. Note that, for illustrative purposes only, $c$ takes negative values.

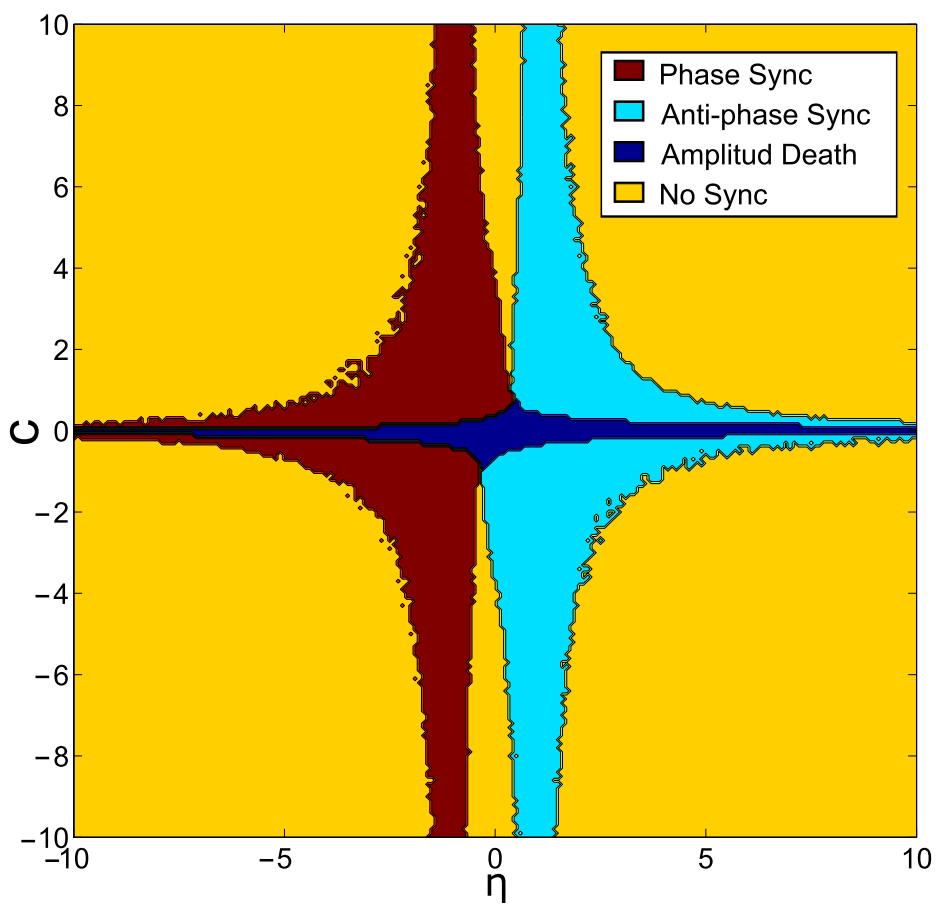

Figure 24. Hyperchaotic synchronization diagram for $c$ with respect to $\eta$ with $b=0.5$.

\section{Conclusions}

In this paper, we presented the emergence of hyperchaos in a network with two very simple discrete periodic oscillators. We emphasized that when the oscillators are isolated, there is certainly no chaos or hyperchaos generated, i.e., the oscillators are structurally non-chaotic. In addition, 
the presented hyperchaotic coupled system synchronizes based on the proposed coupling scheme; this was proven by creating a diagram that shows the synchronization state: in phase or anti-phase. Significantly, these emergent hyperchaotic dynamics can be mainly used in engineering applications, such as cryptography, secure communications, biometric systems, telemedicine, among others; see for example [23,24], where with the purpose of carrying out the experimental implementation, we can use microcontrollers, FPGAs or any other device [26]. In future works, we will perform a theoretical analysis to determine the conditions for the emergence of hyperchaos in networks with discrete periodic oscillators, including networks with a large number of coupled nodes. In this same direction, we will try to show the emergence of hyperchaos in some types of coupled periodic oscillators in real-life networks.

Acknowledgments: This paper was supported by the CONACYT, México under Research Grant 166654.

Author Contributions: Adrian Arellano-Delgado conceived and designed the model; Rosa Martha López-Gutiérrez and Miguel Angel Murillo-Escobar implemented the model and conducted the simulations; Liliana Cardoza-Avendaño wrote the paper; Cesar Cruz-Hernández supervised the work at every stage. All authors have read and approved the final manuscript.

Conflicts of Interest: The authors declare no conflict of interest.

\section{References}

1. Chun-Ni, W.; Ma, J.; Liu, Y.; Huang, L. Chaos control, spiral wave formation, and the emergence of spatiotemporal chaos in networked Chua circuits. Nonlinear Dyn. 2012, 67, 139-146.

2. Courbage, M.; Kazantsev, V.B.; Nekorkin, V.I.; Senneret, M. Emergence of chaotic attractor and anti-synchronization for two coupled monostable neurons. Chaos 2004, 14, 1148-1156.

3. Awrejcewicz, J. Bifurcation and Chaos in Coupled Oscillators; World Scientific Publishing: Teaneck, NJ, USA, 1991.

4. Ulrichs, H.; Mann, A.; Parlitz, U. Synchronization and chaotic dynamics of coupled mechanical metronomes. Chaos 2009, 19, 043120.

5. Kanter, I. Emergence of Chaos in Asymmetric Networks. Phys. Rev. Lett. 1996, 77, 4844-4847.

6. Zhang, H.F.; Wu, R.X.; Fu, X.C. The emergence of chaos in complex dynamical networks. Chaos Solitons Fractals 2006, 28, 472-479.

7. Yuan, W.J.; Luo, X.S.; Jiang, P.Q.; Wang, B.H.; Fang, J.Q. Transition to chaos in small-world dynamical network. Chaos Solitons Fractals 2008, 37, 799-806.

8. Xiang, L.; Guanrong, C.; King-Tim, K. Transition to chaos in complex dynamical networks. Physica A 2008, 338, 367-378.

9. Perlikowski, P.; Yanchuk, S.; Wolfrum, M.; Stefanski, A.; Mosiolek, P.; Kapitaniak, T. Routes to complex dynamics in a ring of unidirectionally coupled systems. Chaos 2010, 20, 013111.

10. Arroyo-Almanza, D.A.; Pisarchik, A.N.; Ruiz-Oliveras, F.R. Route to chaos in a ring of three unidirectionally-coupled semiconductor Lasers. IEEE Photonics Technol. Lett. 2012, 24, 605-607.

11. Arellano-Delgado, A.; López-Gutiérrez, R.M.; Cruz-Hernández, C.; Posadas-Castillo, C.; Cardoza-Avendaño, L.; Serrano-Guerrero, H. Experimental network synchronization via plastic optical fiber. Opt. Fiber Technol. 2013, 19, 93-108.

12. Posadas-Castillo, C.; Cruz-Hernández, C.; López-Gutiérrez, R.M. Experimental realization of synchronization in complex networks with Chua's circuits like nodes. Chaos Solitons Fractals 2007, 40, 1963-1975.

13. Liu, S.; Zhou, L. Network synchronization and application of chaotic Lur'e systems based on event-triggered mechanism. Nonlinear Dyn. 2016, 83, 2497-2507.

14. Chen, D.; Zhang, R.; Ma, X.; Liu, S. Chaotic synchronization and anti-synchronization for a novel class of multiple chaotic systems via a sliding mode control scheme. Nonlinear Dyn. 2012, 69, 35-55.

15. Mirollo, R.E.; Strogatz, S.H. Synchronization of pulse-coupled biological oscillators. SIAM J. Appl. Math. 1990, 50, 1645-1662.

16. Strogatz, S.H. Spontaneous Synchronization in nature. In Proceedings of the International Frequency Control Symposium, Orlando, FL, USA, 30 May 1997.

17. Lewis, F.L.; Zhang, H.; Hengster-Movric, K.; Das, A. Cooperative Control of Multi-Agent Systems Optimal and Adaptive Design Approaches; Springer: London, UK, 2013. 
18. Arellano-Delgado, A.; Cruz-Hernández, C.; López-Gutiérrez, R.M.; Posadas-Castillo, C. Outer synchronization of simple firefly discrete models in coupled networks. Math. Probl. Eng. 2015, 2015, 895379, doi:10.1155/2015/895379.

19. Chen, B.S.; Hsieh, C.Y.; Ho, S.J. System Entropy Measurement of Stochastic Partial Differential Systems. Entropy 2016, 18, 99.

20. Garbaczewski, P. Differential entropy and time. Entropy 2005, 7, 253-299.

21. Harremoës, P.; Topsøe, F. Maximum Entropy Fundamentals. Entropy 2001, 3, 191-226.

22. Gao, J.; Liu, F.; Zhang, J.; Hu, J.; Cao, Y. Information Entropy As a Basic Building Block of Complexity Theory. Entropy 2013, 15, 3396-3418.

23. Cruz-Hernández, C.; López-Gutiérrez, R.M.; Aguilar-Bustos, A.Y.; Posadas-Castillo, C. Communicating encrypted information based on synchronized hyperchaotic maps. Int. J. Nonlinear Sci. Numer. 2010, 11, 337-349.

24. Cruz-Hernández, C.; Aguilar-Bustos, A.Y. Synchronization of discrete-time hyperchaotic systems. CSF 2009, 41, 1301-1310.

25. Song, C.; Qiao, Y. A Novel Image Encryption Algorithm Based on DNA Encoding and Spatiotemporal Chaos. Entropy 2015, 17, 6954-6968.

26. Méndez-Ramírez, R.; Arellano-Delgado, A.; Cruz-Hernández, C.; Martínez-Clark, R. A new simple chaotic Lorenz-type system and its digital realization using a TFT touch-screen display embedded system. Complexity 2107, 2017, 6820492, doi:10.1155/2017/6820492.

27. Wang, X.; Chen, G. Synchronization in small-world dynamical networks. Int. J. Bifurc. Chaos 2002, 12, $187-192$.

28. Wang, X. Complex networks: Topology, dynamics and synchronization. Int. J. Bifurc. Chaos 2002, 12, 885-916.

29. Gottwald, G.A.; Melbourne, I. A new test for chaos in a deterministic system. Proc. R. Soc. A 2004, 460, 603-611.

30. Farmer, J.D.; Ott, E.; Yorke, J.A. The dimension of chaotic attractors. Physica D 1983, 7, 153-180.

31. Wolf, A.; Swift, J.B.; Swinney, H.L.; Vastano, J.A. Determining Lyapunov Exponents from a Time Series. Physica D 1985, 16, 285-317.

32. Theiler, J. Efficient algorithm for estimating the correlation dimension from a set of discrete points. Phys. Rev. A 1987, 36, 4456-4462.

(C) 2017 by the authors. Licensee MDPI, Basel, Switzerland. This article is an open access article distributed under the terms and conditions of the Creative Commons Attribution (CC BY) license (http://creativecommons.org/licenses/by/4.0/). 